\title{
The modelling of the line-locking effect in broad absorption line QSOs
}

\author{
Emmanuil Y. Vilkoviskij and Sergey N. Yefimov \\ Fessenkov Astrophysical Institute, Observatory, 050020 Almaty, Kazakhstan \\ email: vilk@aphi.kz
}

\begin{abstract}
We present a unification model of the matter outflow from AGN. The model includes calculations of the hot gas dynamics, the dynamics of the cold clouds in the hot gas flow, and the radiation transfer in this two-phase medium. We used the model for calculation of the ultraviolet spectrum of the quasar q1303+308. The spectrum of this object is a well-know example of the line-locking effect. Our model calculations permits for the first time to obtain some thin details seen in the observed absorption spectrum, which confirms the validity of the main issues of our theory and model.
\end{abstract}

Keywords. Galaxies: active - (galaxies:) quasars: absorption lines - (galaxies:) quasars: individual (q1303+308) - galaxies: evolution

\section{Introduction}

Our theoretical approach to the problem of matter outflow from AGN was presented in Vilkoviskij \& Karpova (1996), and in complete it was described in Vilkoviskij et al. (1999, 2006).

We suppose that AGN consists of three main subsystems: the central super-massive black hole (SMBH), compact stellar cluster (CSC), and gas subsystem. The AGN evolution, driven by intergalactic interactions and merging (e.g., Sanders et al. (1988); Menci et al. (2003) and the references therein) leads naturally to this structure. The corresponding evolution sequence of an AGN during a "duty cycle" is very well compatible with the standard geometrical unification model (Antonucci 1993).

\section{The unifying model and the line-locking effect}

From the evolution scheme a unifying outflow model follows, which includes both the "classical" AGN1-AGN2 unification and the absorbing outflow models. We suppose that the main absorbing outflow is placed at the internal surface of the obscuring torus. We take into account the dynamics of the hot gas outflow in the summary gravitation field of the massive black hole and compact stellar cluster, the cold cloud dynamics in the hot gas flow (including radiation pressure and drag forces calculations), and the radiation transfer in the clouded media. Our model was tested with the calculations of spectra of different objects, including in particularly the ultraviolet spectrum of the quasar q1303+308.

The line-locking effect appears in our model as the result of non-linear interaction of the radiation pressure and drag force, which leads to a "ladder" velocity structure $V(r)$. The stability of the narrow spectral details in this case means stability of the velocity structure.

The resulting calculated spectrum in comparison to the observed one (Vilkoviskij \& Irwin 2001) is shown in Fig. 1. One can see that it is rather good coincidence in many 


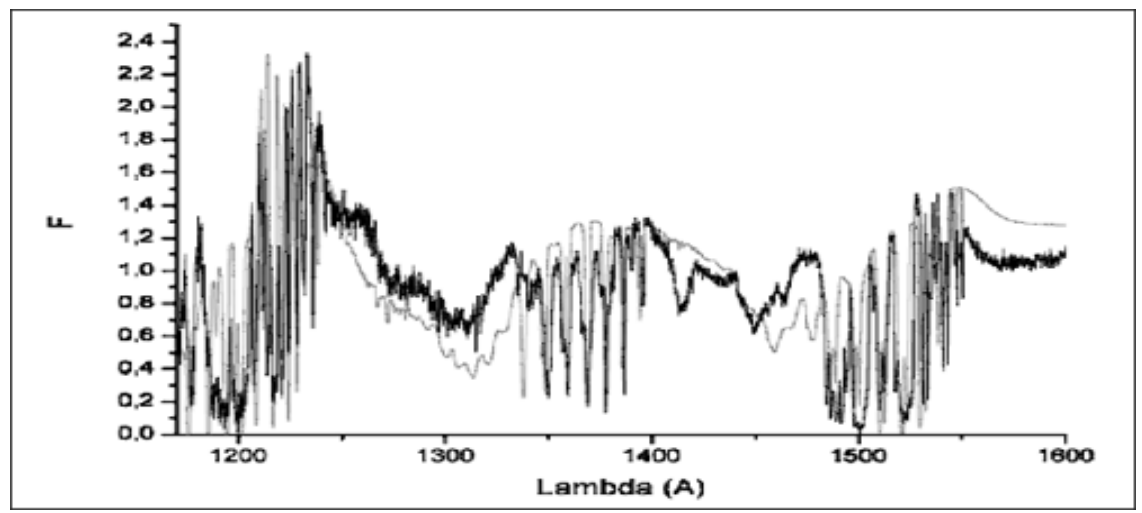

Figure 1. Calculated (gray) and observed (black) spectrum of QSO q1303+308.

details of the calculated and observed spectra (taking into account the model simplifications such as equal masses of cloudlets, some uncertainty of the supposed non-absorbed spectrum, and so on). This proves that our dynamical model explains the line-locking effect.

\section{Conclusions}

Though the numerical dynamical model, which we used for the q1303+308 spectrum simulation, includes some simplifications, it is the only model explaining the observed spectra with the effect of line-locking seen in the UV band of BALQSOs. We call to those observing in X-rays to obtain the spectrum of q1303+308 in this spectral band. The variability of the UV spectrum of the object is of great interest (Foltz et al. 1987, Vilkoviskij \& Irwin 2001). Further observations of this unique object in the optical, UV and X-ray bands are desirable for the sake of deeper understanding of the AGN mass outflow physics.

\section{References}

Antonucci, R. 1993, ARAA, 31, 473

Foltz, C. B., Weymann, R. J., Morris, S .L. \& Turnshek, D. A. 1987, ApJ, 317, 450

Menci, N., Cavaliere, A., Fontana, A., Giallongo, E., Poli, F. \& Vittonini, V. 2003, ApJ, 587, L63

Sanders, D. B., Soifer, B .T., Elias, J. H., Neugebauer, G. \& Matthews, K. 1988, ApJ, 328, L35

Vilkoviskij, E. Y., Efimov, S. N., Karpova, O. G. \& Pavlova, L. A. 1999, MNRAS, 309, 80

Vilkoviskij, E. Y. \& Irwin, M. J. 2001, MNRAS, 321, 4

Vilkoviskij, E. Y. \& Karpova, O. G. 1996, PAZ, 22, 148

Vilkoviskij, E. Y., Lovelace, R. V. E., Pavlova, L. A., Romanova, M. M. \& Yefimov, S. N. 2006, Ap\&SS, 306, 129 